Pacific Journal of Mathematics

THE ABEL-JACOBI ISOMORPHISM FOR THE CUBIC
FIVEFOLD 


\title{
THE ABEL-JACOBI ISOMORPHISM FOR THE CUBIC FIVEFOLD
}

\author{
Alberto Collino
}

Let $X$ be a general cubic fivefold, $J X$ the associated intermediate Jacobian, $F$ the Fano surface of the planes contained in $X$. We prove that the Abel-Jacobi map induces an isomorphism from the Albanese variety of $F$ to $J X$.

Introduction. It is a standard fact (see [7] Exp. XI (2.9)) that the only smooth hypersurfaces $X$ in $\mathbf{P}^{2 d+1}, d>0$, for which the intermediate Jacobian $J X$ is an abelian variety are the quadrics, the cubic and the quartic threefolds in $\mathbf{P}^{4}$, and the cubic fivefold in $\mathbf{P}^{6}$. For a quadric $J X=0$. In [5], Clemens and Griffiths proved that the Abel-Jacobi map

$a: \mathrm{Alb} F \rightarrow J X$

is an isomorphism, where $X$ is the smooth cubic threefold and $F$ is the (smooth) Fano surface of the lines on $X$. Recently Letizia, [9], using a method which he credits Clemens for, [4], proved that $(+)$ is an isomorphism also when $X$ is a general smooth quartic threefold and $F$ is the Fano surface of the conics on $X$.

Here we complete the picture, proving that $(+)$ is an isomorphism also when $X$ is a general smooth cubic fivefold, $F$ being the surface of the planes on $X$. Our tool is the Clemens-Letizia method coupled with some ideal which originated from [6].

We work with varieties defined over the complex numbers field.

(I). Let $T$ be a plane in $\mathbf{P}^{6}$ and let $X$ be a cubic hypersurface containing it. We choose projective coordinates $\left(x_{0}: x_{1}: x_{2}: \cdots: x_{6}\right)$ in $\mathbf{P}^{6}$ so that $T$ has equations $x_{0}=x_{1}=x_{2}=x_{3}=0$. The equation of $X$ is then of the form

$$
\begin{aligned}
0= & Q_{0} x_{0}+Q_{1} x_{1}+Q_{2} x_{2}+Q_{3} x_{3}+\sum\left(A_{i j k} x_{i} x_{j} x_{k}\right) \\
& +B\left(x_{0}, x_{1}, x_{2}, x_{3}\right)
\end{aligned}
$$

where $4 \leq k \leq 6,0 \leq i \leq j \leq 3, B$ is homogeneous cubic and $Q_{i}, i=$ $0, \ldots, 3$, are homogeneous polynomials of degree 2 in $x_{4}, x_{5}, x_{6}$. 
Let $C_{i}$ be the conic on $T$ of equation $Q_{i}=0, X$ is non-singular along $T$ if and only if $\cap C_{i}=\varnothing$. In the following we shall assume that $X$ is smooth along $T$, when it is not explicitly otherwise stated. We shall denote $F(X)$, or simply $F$, the variety of planes contained in $X$; more precisely we take $F$ to be the Hilbert scheme of the two-dimensional planes of $X$, [1]. In order to study $F$ we need to compute $H^{0}(T, N(T, X))$, which is the tangent space to $F$ at the point $t$ representing $T$. When there is no confusion we shall write $N$ for the normal bundle $N(T, X)$ and $h^{0}(N)=$ $\operatorname{dim} H^{0}(T, N)$.

(1.2) Proposition. $h^{0}(N)=2 \leftrightarrow C_{0}, C_{1}, C_{2}, C_{3}$, are linearly independent.

Proof. We start from the exact sequence of sheaves on $T$

$$
0 \rightarrow N \rightarrow O(1)^{\oplus 4} \stackrel{f}{\rightarrow} O(3) \rightarrow 0,
$$

where the middle sheaf is $N\left(T, \mathbf{P}^{6}\right)$ and $O(3)$ is $\left.N\left(X, \mathbf{P}^{6}\right)\right|_{T}$. If one chooses conveniently the splitting of $N\left(T, \mathbf{P}^{6}\right)$ then $f$ is given by the matrix $\left(Q_{0}\right.$, $Q_{1}, Q_{2}, Q_{3}$ ), hence: (a) $h^{0}(N)=2 \leftrightarrow$ (b) $f: H^{0}\left(T, O(1)^{\oplus 4}\right) \rightarrow H^{0}(T, O(3))$ is surjective $\leftrightarrow$ (c) given any homogeneous cubic polynomial $K\left(x_{4}, x_{5}, x_{6}\right)$ there are linear homogeneous polynomials $L_{0}, L_{1}, L_{2}, L_{3}$ such that (+): $K=\sum L_{i} Q_{i}$.

If the $C_{i}$ are linearly dependent then $f$ cannot be surjective on global sections, because the space of polynomials $K$ in $(+)$ has dimension 9 at most. We assume now that the $C_{i}$ are linearly independent. Since the four conics have no common point, the general member of the web they span is non singular; without restriction we may assume that $C_{1}$ is smooth, so that $C_{1} \cap C_{2}$ is finite and we may take $C_{3}$ in such a way that $C_{1} \cap C_{2} \cap C_{3}=$ $\varnothing$. In the ring $R=k\left[x_{4}, x_{5}, x_{6}\right]$ we let $I=$ the ideal $\left(Q_{1}, Q_{2}, Q_{3}\right)$, $R_{3}=$ the vector space of homogeneous cubic polynomials, $I_{3}=R_{3} \cap I$. By a theorem of Macaulay, [8], the pairing $g:(R / I) \times(R / I) \rightarrow\left(R_{3} / I_{3}\right)$ $\simeq \mathbf{C}$ is a perfect duality, where $g$ is given by the product of representative of equivalence classes. It follows:

(i) $I_{3}$ has codimension 1 in $R_{3}$, (ii) given $Q_{0}$, since $Q_{0} \notin I$ by hypothesis, there is $L_{0}$ such that $g\left(L_{0} Q_{0}\right) \neq 0$, i.e. $L_{0} Q_{0} \notin I_{3}$. Therefore $I_{3}+\left(L_{0} Q_{0}\right)=R_{3}$, hence $f$ is surjective on global sections.

(1.4) COROLLARY. $F$ is a non-singular surface at the point $t$ representing $T$ if and only if $C_{0}, \ldots, C_{3}$ are linearly independent. 
Proof. $h^{1}(N)=0 \leftrightarrow h^{0}(N)=2 \leftrightarrow\left(C_{0}, \ldots, C_{3}\right)$ are linearly independent.

We recall that in the preceding corollary we had the tacit assumption that $X$ was smooth along $T$. For next definition we drop it.

(1.5) Definition. Let $T$ be a plane contained in $X$; using the notations above we say that $T$ is a special plane for $X$, or that $X$ is special with respect to $T$, if $C_{0}, \ldots, C_{3}$ are linearly dependent.

If $X$ is non-singular along $T$ then $F$ is singular at $t$ if and only if $T$ is special; if $X$ has an ordinary node on $T$ we shall see that $F$ is not normal at $t$, but we shall also see that if $T$ is not special then on the normalization $F^{+}$of $F$ the points $t_{1}$ and $t_{2}$, which map to $t$, are non-singular.

Let $H$ be the Hilbert scheme parametrizing the totality of cubic hypersurfaces of $\mathbf{P}^{6}, H$ is naturally isomorphic to $\mathbf{P}^{83}$. We let $H^{s}=$ the subvariety of $H$ of the cubics with special planes, $D=$ the subvariety of the singular cubics.

(1.6) Lemma. (a) $\operatorname{cod}\left(H^{s}, H\right) \geq 1$, (b) $H^{s}$ is irreducible and $H^{s} \not \subset D$.

Proof. Let $H^{T} \simeq \mathbf{P}^{73}$ be the variety of the cubics containing $T$. Keeping the notations above (1.1) we note that $X$ is special along $T$ if there are $b_{0}, \ldots, b_{3}$ with $(+): \sum b_{i} Q_{i}=0$. Let $\mathbf{A}^{24}$ be the affine space in the variables $a_{i j}^{k}$, the coefficients of the $Q_{k}$ 's. Let $\left(b_{0}, \ldots, b_{3}\right)$ be homogeneous coordinates for $\mathbf{P}^{3}$. Condition (+) gives six bilinear equations in $\mathbf{P}^{3} \times \mathbf{A}^{24}$, let $V$ be the determined variety. It is easy to see that $V \rightarrow \mathbf{P}^{3}$ is an $\mathbf{A}^{18}$ fibration, further the projection $V \rightarrow \operatorname{pr}(V) \hookrightarrow \mathrm{A}^{24}$ is birational to the image $\operatorname{pr}(V)$, therefore $\operatorname{pr}(V)$ is irreducible with $\operatorname{dim}=21$. Let $H^{s, T}$ be the variety of the cubics which are special along $T$, because of our remark $\operatorname{cod}\left(H^{s, T}, H^{T}\right)=3$ and $H^{s, T}$ is irreducible. Let $G=G(2,6)$ be the grassmannian of the planes in $\mathbf{P}^{6}$, then $H^{s}=\bigcup_{T \in G}\left(H^{s, T}\right)$, so that $H^{s}$ is irreducible and $\operatorname{dim} H^{s} \leq 82$. In order to see that $H^{s} \not \subset D$ it suffices now to produce one cubic which is non-singular and contains a special plane. The Fermat cubic $\sum x_{i}^{3}=0$ has this property: let $r$ be a third root of -1 , i.e. $r^{3}=-1$, change coordinates $z_{0}=x_{0}-r x_{5}, z_{1}=x_{1}-r x_{6}, z_{2}=x_{2}$, $z_{3}=x_{3}-r x_{4}, z_{4}=x_{4}, z_{5}=x_{5}, z_{6}=x_{y}$, it is easy to see that the plane $z_{0}=z_{1}=z_{2}=z_{3}$ is special for this cubic. 
(1.7) in $G \times H$ we set $I=\{(T, X): T \subseteq X\}, I$ is the incidence correspondence; $I$ is a $\mathbf{P}^{73}$ fibre bundle over $G$, so that $\operatorname{dim} I=85$. Let $p$ : $I \rightarrow G$ and $q: I \rightarrow H$ be the projections, the fibre $q^{-1}(x)=F(X)$, the Hilbert scheme of the planes contained in $X$, the hypersurface represented by $x$. Fix now a point $(T, X)$ in $I$ such that $T$ is non-special for $X$ and $X$ is smooth along $T$ (it follows from the proof of the lemma that there is such a couple) then $F(X)$ is a smooth surface at the point $t$ representing $T$. Then the general $F(X)$ is a surface, non-singular because it does not contain special planes, moreover it is irreducible because of

(1.8) Proposition. For all $X, F(X)$ is connected.

Proof. Following an idea of Barth and Van de Ven [2] we need only to check that the set $S=\{(T, X): F(X)$ is not a smooth surface at $T\}$ has codimension at least 2 in $p^{-1}(T)$. Now $F(X)$ is not a smooth surface at $T$ only if either $T$ is special for $X$ or if $X$ is singular at some points of $T$. The first case is covered by the proof of (1.6), indeed $p^{-1}(T)=H^{T}$ and we proved $\operatorname{cod}\left(H^{s, T}, H^{T}\right)=3$. On the other hand let $D^{T}=\left\{X \in H^{T}: X\right.$ is singular at some point of $T\}$, by a similar argument as for $H^{s, T}$ one can see $\operatorname{cod}\left(D^{T}, H^{T}\right)=2$.

(1.9) Remark. In $G \times H^{s}$ let $I^{s}=\{(T, X): T$ is special for $X\}$; it follows from the proof of (1.6) that $\operatorname{dim} I^{s}=82$, hence either (a) $\operatorname{dim} H^{s}$ $=82$, so that in a general cubic which is special there is a finite number of special planes, or (b) $\operatorname{dim} H^{s}<82$, so that given a general pencil of cubics none of the cubics is special.

Collecting the preceding results we see

(1.10) Proposition. Let $\left\{X_{t}\right\}, t \in \mathbf{P}^{1}$, be a general Lefschetz pencil of cubic fivefolds, let $\left\{F\left(X_{t}\right)\right\}, t \in \mathbf{P}^{1}$, be the associated family of Fano varieties, let $t_{1}, \ldots, t_{N}$ be such that $X_{t}$ is smooth for $t \in \mathbf{P}^{1}-\left\{t_{1}, \ldots, t_{N}\right\}$. Then there exists $t_{N+1}, \ldots, t_{N+M}$ in $\mathbf{P}^{1}$ such that:

(1) $F\left(X_{t}\right)$ is a smooth and irreducible surface for $t \in \mathbf{P}^{1}-\left\{t_{1}, \ldots, t_{N}\right.$, $\left.t_{N+1}, \ldots, t_{N+m}\right\}$.

(2) The surface $F\left(X_{t_{N+J}}\right)$ has only isolated singularities, $1 \leq J \leq M$.

(3) The surface $F\left(X_{t_{j}}\right)$ has as only singularities the locus of the planes through the ordinary double point of $X_{t}, 1 \leq J \leq N$. 
In order to complete the program according to the Clemens-Letizia method [9] we still need to check that the Abel-Jacobi map $F \rightarrow J X$ is not constant, and also to prove the following

(1.11) THEOREM. Let $F_{0}$ be the Fano surface of the planes on $X_{0}$, general cubic hypersurface with one single singular point $p_{0}$ which is an ordinary node. Then the family $D$ which represents the planes through $p_{0}$ is a smooth irreducible curve; $F_{0}-D$ is non-singular; along $D F_{0}$ is analytically reducible in two smooth components meeting transversally.

(II). This section is devoted to the proof of Theorem (1.11). We need some preliminary considerations.

Let $b: \mathbf{P}^{+} \rightarrow \mathbf{P}^{6}$ be the blow up of $\mathbf{P}^{6}$ at $p_{0}$, let $E=b^{-1}\left(p_{0}\right)$ be the exceptional divisor, let $X^{+}$be the strict transform of $X_{0}$, let $Q=X^{+} \cap E$ be the exceptional quadric. As in [5] the linear projection $X_{0} \rightarrow \mathbf{P}^{5}$ of centre $p_{0}$ induces a birational morphism $\lambda: X^{+} \rightarrow \mathbf{P}^{\prime}$, which turns out to be the blow up of $\mathbf{P}^{5}$ along the threefold $Y$, the $(2,3)$ complete intersection of $Q$ with a cubic $K$. More precisely let $p_{0}=(0, \ldots, 0,1)$, let $\mathbf{P}^{5}$ be the hyperplane $x_{6}=0$, then the cubic $X_{0}$ has equation

$$
0=Q\left(x_{0}, x_{1}, \ldots, x_{5}\right) x_{6}+K\left(x_{0}, \ldots, x_{5}\right)
$$

and in $\mathbf{P}^{5}$ the cubic and the quadric have equations $K=0$ and $Q=0$. The planes through $p_{0}$ are mapped via $\lambda$ to the lines lying in $Y$ and conversely; hence the family of planes through $x_{0}$ is in general a smooth irreducible curve, because such a curve is the family of the lines in a general $Y$, see [3].

(2.2) Let $T$ be a plane in $\mathbf{P}^{6}$, if $p_{0} \notin T$ then the total transform $b^{-1}(T)$ is isomorphic to $T$ and it is also the proper transform $T^{b}$ of $T$. If $p_{0} \in T$ then the proper transform $T^{b}$ of $T$ is the blowing up of $T$ at $p_{0}$. In this case $T^{b}$ is not the correct transform with respect to the behaviour of the Hilbert scheme; in fact in both cases $T^{b}$ is a complete intersection in $\mathbf{P}^{+}$, but of different type.

(2.3) Definition. We say that a variety $Z$ in $\mathbf{P}^{+}$is a strict biplane if $Z=T^{b} \cup B$, where $B$ is a plane in $E, B \cap T^{b}=L$, where $L$ is the exceptional line in $T^{b}$, proper transform of a plane $T$ through $p_{0}$.

It is easy to see that a strict-biplane $Z$ is the same kind of complete intersection in $\mathbf{P}^{+}$as it is a 'plane' $T^{b}$ not meeting $E$. In the following we denote $G^{+}=$the Hilbert scheme of $P^{+}$of the complete intersections of the same type as a strict biplane. If $a \in G^{+}$we let $Z_{a}$ be the represented 
scheme; we call $Z_{a}$ a biplane and remark that there are only two possibilities: either $Z_{a}$ is a strict biplane or $Z_{a}=T^{b}$, where $T$ is a plane not containing $p_{0}$. One can see easily that $G^{+}$is non-singular of dimension 12 , by explicitly computing the dimension of the tangent space to $G^{+}$at a point representing a given biplane.

There is a more intuitive way to describe $G^{+}$. Let $S$ be the Schubert variety of the planes in $\mathbf{P}^{6}$ through $p_{0}$, then $S$ is smooth, being isomorphic to $G(1,5)$. Let $\beta^{*}: G^{*} \rightarrow G$ be the blow up of $G=G(2,6)$ along $S$. We have $G^{*}=G^{+}$. In fact there is a correspondence $\lambda^{\prime}: G \rightarrow G(2,5)$ obtained by sending a plane from $\mathbf{P}^{6}$ to $\mathbf{P}^{5}$ by means of the linear projection $\lambda$ of centre $p_{0}$. The indeterminacy of $\lambda^{\prime}$ at $S$ is solved by blowing up $G$, so to have $\lambda^{*}: G^{*} \rightarrow G(2,5)$. Via $\left(\beta^{*}, \lambda^{*}\right) G^{*}$ embeds in $G \times G(2,5)$. Similarly there is a map $\beta^{+}: G^{+} \rightarrow G$ and a map $\lambda^{+}: G^{+} \rightarrow G(2,5)$, obtained by setting $\beta^{+}(a)=$ the point representing $b\left(Z_{a}\right), \lambda^{+}(a)=$ the point representing $\lambda\left(Z_{a}\right)$. Also $G^{+}$embeds in $G \times G(2,5)$ and it has the same image as $G^{*}$ has. In this way we get a 1-1 correspondence between $G^{*}$ and $G^{+}$; since they are both non-singular, then they are isomorphic.

We write $F^{+}=$the Fano scheme of the biplanes contained in $X^{+}$and denote $\beta^{+}: F^{+} \rightarrow F$ the map induced by restriction of $\beta^{+}: G^{+} \rightarrow G$. Collecting previous remarks we note

(2.4) Lemma. (1) $\beta^{+}: F^{+}-\beta^{-1}(D) \rightarrow F-D$ is $1-1$. (2) $\left(\beta^{+}\right)^{-1}(D)=$ $D_{1} \cup D_{2}$ where $D_{1}$ and $D_{2}$ are isomorphic to $D$ via $\beta^{+}, D_{1} \cap D_{2}=\varnothing$.

Proof. (1) is clear. For (2) let $t \in D$, then $a \in\left(\beta^{+}\right)^{-1}(t)$ means $Z_{a} \hookrightarrow X, b\left(Z_{a}\right)=T_{t}$ contains $p_{0}, \lambda\left(Z_{a}\right)$ is a plane $B_{a}$ contained in the quadric $Q$ and passing through the exceptional line $L$ in the proper transform $T_{t}^{b}$. In other words $Z_{a}=T_{t}^{b}+B_{a}$. Since $Q$ is a four dimensional smooth quadric then for a fixed $L$ in $Q$ there are only two possible choices for $B_{a}$, one for each system of planes. Statement (2) follows easily.

\section{Our next step is}

(2.5) THEOREM. Let $t$ represent the plane $T$, if $X_{0}$ is not special at $T$ then $F^{+}$is non singular at the points of $\left(\beta^{+}\right)^{-1}(t)$.

If $X$ is smooth along $T$ the theorem is Corollary (1.4). We assume therefore that $p_{0} \in T$ and let $\left(\beta^{+}\right)^{-1}(t)=\{a, b\}, a \in D_{1}, b \in D_{2}$. We denote $N=N\left(Z_{a}, X\right)$ the dual of the conormal bundle of $Z_{a}$ in $X$, our 
program is to prove $h^{0}(N)=2$, hence $F^{+}$is a smooth surface at the point a.

For simplicity we write $T_{t}^{b}=A$, so that $A \cup B=Z_{a}$ and further we set $Z=Z_{a}$. The standard exact sequences of "normal" bundles for the triple $\left(Z, X^{+}, \mathbf{P}^{+}\right)$is

$$
\left.0 \rightarrow N\left(Z, X^{+}\right) \rightarrow N\left(Z, \mathbf{P}^{+}\right) \stackrel{f}{\rightarrow} N\left(X^{+}, \mathbf{P}\right)\right|_{Z} \rightarrow 0 .
$$

(2.7) Let $D_{0}, D_{1}, D_{2}, D_{3}$ be four hypersurfaces in $\mathbf{P}^{+}$which intersect completely in $Z$, then

$$
N\left(Z, \mathbf{P}^{+}\right)=\left.\left.\left.\left.O\left(D_{0}\right)\right|_{Z} \oplus O\left(D_{1}\right)\right|_{Z} \oplus O\left(D_{2}\right)\right|_{Z} \oplus O\left(D_{3}\right)\right|_{Z} .
$$

In the following (2.9) we fix such a splitting so that the restrictions of sequence (2.6) to $A, B, L$ are respectively

$$
\begin{aligned}
& \left(s_{A}\right) \quad 0 \quad \rightarrow \quad N_{A} \quad \rightarrow \quad \oplus^{3} O_{A}(H-L) \oplus O_{A}(H) \stackrel{f_{A}}{\rightarrow} \quad O_{A}(3 H-2 L) \quad \rightarrow \quad 0 \\
& \left(s_{B}\right) \quad 0 \quad \rightarrow \quad N_{B} \quad \rightarrow \quad \oplus^{3} O_{B}(1) \oplus O_{B} \quad \stackrel{f_{B}}{\rightarrow} \quad O_{B}(2) \quad \rightarrow \quad 0 \\
& \left(s_{L}\right) \quad 0 \quad \rightarrow \quad N_{L} \quad \rightarrow \quad \oplus^{3} O_{L}(1) \oplus O_{L} \quad \stackrel{f_{L}}{\rightarrow} \quad O_{L}(2) \quad \rightarrow \quad 0 .
\end{aligned}
$$

Here $H$ is the divisor on $A$ which is the total transform of the line in $T, L$ is the exceptional line in $A . N_{A}, N_{B}$ and $N_{L}$ are the restrictions of $N$ to $A$, $B, L$.

(2.8) There is a standard Mayer-Vietoris sequence

$$
0 \rightarrow H^{0}(Z, N) \rightarrow H^{0}\left(A, N_{A}\right) \oplus H^{0}\left(B, N_{B}\right) \rightarrow H^{0}\left(L, N_{L}\right) ;
$$

in order to prove $h^{0}(Z, N)=2$ we shall prove: (i) $H^{0}\left(B, N_{B}\right) \stackrel{\sim}{\rightarrow}$ $H^{0}\left(L, N_{L}\right)$, (ii) $h^{0}\left(A, N_{A}\right)=2$. We need first to compute $f_{A}, f_{B}, f_{L}$.

(2.9) Looking at the sequences above we remark that $f_{A}$ is a global section in $H^{0}\left(A, \oplus^{3} O_{A}(2 H-L) \oplus O_{A}(2 H-2 L)\right), f_{B}$ is a global section in $H^{0}\left(B, \oplus^{3} O_{B}(1) \oplus O_{B}(2)\right)$, and $f_{L}$ is in $H^{0}\left(L, \oplus^{3} O_{L}(1) \oplus O_{L}(2)\right)$. To compute $f_{A}, f_{B}, f_{L}$ means to identify them as sections of the indicated sheaves, in particular both $f_{A}$ and $f_{B}$ are determined if we find their restrictions to $A-L$ and $B-L$ respectively.

We let $\left(x_{0}, \ldots, x_{6} ; y_{0}, \ldots, y_{5}\right)$ be the bihomogeneous coordinates of $\mathbf{P}^{6} \times \mathbf{P}^{5}$, then $\mathbf{P}^{+}$is the subvariety determined by the equations $x_{l} y_{J}=$ $x_{J} y_{i}, 0 \leq i, j \leq 5$. The biplane $Z$ is the complete intersection in $\mathbf{P}^{+}$of 
equations $y_{0}=y_{1}=y_{2}=x_{3}=0$. We let $D_{i}=\operatorname{locus}\left(y_{i}=0\right) \quad i=0,1,2$; $D_{3}=\operatorname{locus}\left(x_{3}=0\right)$, cf. (2.7). If $f_{A-L}$ denotes the restriction of $f_{A}$ to $A-L=T-p_{0}$, then the same proof of (1.2) gives

$$
f_{A-L}=\left.\left(Q_{0}, Q_{1}, Q_{2}, Q_{3}\right)\right|_{A-L} .
$$

Let $C_{i}^{-}$be the proper transform in $A$ of the conic $C_{i}$ and let $f_{A}=$ $\left(f_{A}^{0}, f_{A}^{1}, f_{A}^{2}, f_{A}^{3}\right)$. We shall see below that one can choose the coordinates $x_{0}, \ldots, x_{3}$ so that both $C_{0}$ and $C_{3}$ have a double point in $p_{0}$, while $C_{1}$ and $C_{2}$ are smooth there. From this and (2.10) it follows

(2.11) Proposition. $\left(C_{0}^{-}+L\right)$ is the divisor of the zeros of $f_{A}^{0}, C_{1}^{-}$of $f_{A}^{1}, C_{2}^{-}$of $f_{A}^{2}, C_{3}^{-}$of $f_{A}^{3}$.

With the notations above, the equations of the plane $B$ in the exceptional divisor $E$ of $\mathbf{P}^{+}$are $y_{0}=y_{1}=y_{2}=0$, while the equations of $T$ in $\mathbf{P}^{6}$ are as before $x_{0}=x_{1}=x_{2}=x_{3}=0$. Since $p_{0}$ is a node the equations of the conics $C_{i}$ are of type

$$
Q_{i}=L_{i}\left(x_{4}, x_{5}\right) x_{6}+Q_{i}^{0}\left(x_{4}, x_{5}\right)
$$

cf. (1.1). In $E$ the exceptional quadric $Q$ of $X^{+}$is therefore

$$
\begin{array}{r}
y_{0} L_{0}\left(y_{4}, y_{5}\right)+y_{1} L_{1}\left(y_{4}, y_{5}\right)+y_{2} L_{2}\left(y_{4}, y_{5}\right)+y_{3} L_{3}\left(y_{4}, y_{5}\right) \\
+\sum\left(A_{i j 6} y_{i} y_{j}\right)=0, \quad 0 \leq i, j \leq 3 .
\end{array}
$$

Since $B$ is contained in $Q$ one has

$$
A_{336}=0, \quad L_{3}\left(y_{4}, y_{5}\right)=0
$$

so that $C_{3}$ has a node in $p_{0}$. Next we use the hypothesis of the linear independence of the $C_{i}$ to remark that, up to a linear change in $x_{0}, x_{1}, x_{2}$, one may assume that also $C_{0}$ has a node in $p_{0}$, i.e. $L_{0}\left(x_{4}, x_{5}\right)=0$. Now we recall that the exceptional line $L$ has equations $y_{0}=y_{1}=y_{2}=y_{3}=0$ in $E$ and that we have $Z_{a}=A+B$ with $B=B_{a}$ and also $Z_{b}=A+B_{b}$, where $A \cap B_{b}=L=A \cap B$. Without restriction we may require that $B_{b}$ has equations $y_{1}=y_{2}=y_{3}=0$. The equation of $Q$ in $E$ is then

$$
\begin{aligned}
& y_{1} L_{1}\left(y_{4}, y_{5}\right)+y_{2} L_{2}\left(y_{4}, y_{5}\right)+\sum A_{i j 6} y_{i} y_{j}, \\
& \text { with } 0 \leq i \leq j \leq 3 \text {, and } A_{006}=A_{336}=0 .
\end{aligned}
$$

By hypothesis $Q$ is of maximal rank, then by a linear change of coordinates we may assume $L_{1}\left(y_{4}, y_{5}\right)=y_{4}, L_{2}\left(y_{4}, y_{5}\right)=y_{5}$ and also note that $A_{036} \neq 0$. 
(2.16) COROLlaRY.

$$
f_{B}=\left(A_{036} y_{3}, y_{4}+A_{136} y_{3}, y_{5}+A_{236} y_{3}, Q_{3}^{0}\left(y_{4}, y_{5}\right)+(\cdots) y_{3}\right)
$$

(2.17) CoRollary. $f_{L}=\left(0, y_{4}, y_{5}, Q_{3}^{0}\left(y_{4}, y_{5}\right)\right)$.

Proof (2.16). We just outline the computation. It suffices to compute the restriction of $f_{B}$ to the affine plane $B-L$ (i.e. the locus $y_{3} \neq 0$ ), so we restrict everything to the affine space $V$ which is the locus in $\mathbf{P}^{+}$where $y_{3} \neq 0, x_{6} \neq 0$. There $B-L$ is the complete intersection $y_{0}=y_{1}=y_{2}=$ $x_{3}=0$. Now in $V y_{0}, y_{1}, y_{2}, x_{3}, y_{4}, y_{5}$ induce natural linear parameters which we write $y_{0}^{0}, y_{1}^{0}, y_{2}^{0}, x_{3}^{0}, y_{4}^{0}, y_{5}^{0}$. The equation of the restriction of $X^{+}$ to $V$ is then of the form $M_{0}() y_{0}^{0}+M_{1}() y_{1}^{0}+M_{2}() y_{2}^{0}+M_{3}() x_{3}^{0}=0$. It follows that the restriction of $f_{B}$ to $B-L$ is equal to the restriction of $\left(M_{0}, M_{1}, M_{2}, M_{3}\right)$. An explicit computation of the $M_{i}$ 's yields the result.

(2.18) Corollary. $h^{0}\left(B, N_{B}\right)=h^{0}\left(L, N_{L}\right)=4$.

Proof. Obvious since $A_{036} \neq 0$.

Let $g: H^{0}\left(B, N_{B}\right) \rightarrow H^{0}\left(L, N_{L}\right)$ be the restriction map; using the short exact sequences of global sections associated with $s_{B}$ and $s_{L}$ and the snake lemma one finds

$$
\operatorname{Ker}(g)=\operatorname{Ker}\left(h: \oplus^{3} H^{0}(B, 0) \oplus H^{0}(B, 0(-1)) \rightarrow H^{0}(B, 0(1))\right)
$$

where the matrix of $h$ is just the matrix of $f_{B}$. So $h$ is surjective and $g$ and $h$ are both isomorphisms.

(2.19) Corollary. $g: H^{0}\left(B, N_{B}\right) \stackrel{\sim}{\rightarrow} H^{0}\left(L, N_{L}\right)$.

We have proved part (i) in (2.8); next we show

(2.20) Proposition. If $Q_{0}, Q_{1}, Q_{2}, Q_{3}$ are linearly independent then $h^{0}\left(A, N_{A}\right)=2$.

Proof. Looking at the long exact sequence of cohomology associated with $s_{A}$ we see $h^{0}\left(A, N_{A}\right)=2 \leftrightarrow h^{1}\left(A, N_{A}\right)=0 \leftrightarrow f_{A}$ is surjective on global sections. Let $P\left(x_{4}, x_{5}, x_{6}\right)=W\left(x_{4}, x_{5}\right) x_{6}+V\left(x_{4}, x_{5}\right)$ be a cubic polynomial, to prove that $f_{A}$ is surjective amounts to produce $L_{0}\left(x_{4}, x_{5}\right)$, 
$L_{1}\left(x_{4}, x_{5}\right), L_{2}\left(x_{4}, x_{5}\right), L_{3}\left(x_{4}, x_{5}, x_{6}\right)$ linear polynomials in the indicated variables so that $\sum L_{i} Q_{i}=P$ where $Q_{i}$ are the quadrics in (2.12). For later use we note that we shall in fact produce $L_{3}\left(x_{4}, x_{5}\right)$.

Using the simplifications established above we have $Q_{0}=A B, Q_{1}=$ $x_{4} x_{6}+F G, Q_{2}=x_{5} x_{6}+D E, Q_{3}=C H$ with $A, B, \ldots, H$ linear homogeneous polynomials in $x_{4}, x_{5}$. Using the hypothesis of the linear independence of $Q_{0}, \ldots, Q_{3}$ we can assume $F G=0$, up to a linear change of coordinates $x_{4}, x_{5}$. We notice first that there are $L_{1}$ and $L_{2}$ such that $L_{1} x_{4}+L_{2} x_{5}=W\left(x_{4}, x_{5}\right)$ and that also $L_{1}^{\prime}=L_{1}-\alpha x_{5}, L_{2}^{\prime}=L_{2}+\alpha x_{4}$ satisfy the equation. So we need to find $L_{0}\left(x_{4}, x_{5}\right), L_{3}\left(x_{4}, x_{5}\right)$ and a constant $\alpha$ such that

$$
L_{0} A B+\left(L_{2}+\alpha x_{4}\right) D E+L_{3} C H=V\left(x_{4}, x_{5}\right) .
$$

Equivalently for any $U\left(x_{4}, x_{5}\right)$ we look for $L_{0}, L_{3}, \alpha$ such that $(++)$

$$
L_{0} A B+\alpha x_{4} D E+L_{3} C H=U .
$$

in other words we want to show that the dimension of the vector space of polynomials in $(++)$ is 4 . If $A B$ and $C H$ have no common factor a solution for $(++)$ exists with $\alpha=0$, because of the theorem of Macaulay. If $A B$ and $C H$ have a common factor then it can be only a linear factor, because $A B=Q_{0}$ and $C H=Q_{3}$ are linearly independent by hypothesis. We assume then that $B=H$ and also that $A$ and $C$ are not proportional. In this case the linear system $\left\{\left(L_{0} A+L_{3} C\right) B\right\}$ has dimension 3 and the system $\left\{L_{0} A B+L_{3} C H+\alpha x_{4} D E\right\}$ has dimension 4 if $x_{4} D E \notin$ $\left\{\left(L_{0} A+L_{3} C\right) B\right\}$; we need therefore to exclude that either (i) $x_{4}$ or (ii) $D$ or (iii) $E$ is proportional to $B$. In case (iii) or (ii) the point $x_{6}=$ $B=0$ on $T$ is a point in the intersection of the conics $C_{i}$, hence it is a second singular point on $X_{0}$, which is a contradiction. In case (i) similarly the set $x_{4}=Q_{2}=0$ contains another singular point on $X_{0}$, again a contradiction.

(2.21) In order the complete the proof of (1.11) we show below that the differential of $\beta^{+}$at the point $a$ is injective and next that if $a$ and $b$ are the two points in the fibre $\left(\beta^{+}\right)^{-1}(t)$ then

$$
\operatorname{dim}\left(d \beta^{+}\left(T_{a}\left(F^{+}\right)\right) \cap d \beta^{+}\left(T_{b}\left(F^{+}\right)\right)\right)=1 .
$$

Proposition. $d \beta_{a}^{+}: T_{a}\left(F^{+}\right) \rightarrow T_{\beta+(a)}(G)$ is injective.

Proof. Recall $T_{a}\left(F^{+}\right)=H^{0}\left(Z_{a}, N\right)=H^{0}\left(A, N_{a}\right)$. From the long sequence of cohomology of the sequence $\left(s_{A}\right)$ we get the upper exact row in the following diagram. 
$\left(D_{a}\right)$ :

$$
\begin{gathered}
0 \rightarrow T_{a}\left(F^{+}\right) \stackrel{i_{a}}{\rightarrow} H^{0}(A, O(H-E) \oplus O(H-E) \oplus O(H-E) \oplus O(H)) \stackrel{f_{A}}{\rightarrow} H^{0}(A, O(3 H-2 E)) \rightarrow 0 \\
H^{0}(T, O(H) \oplus O(H) \oplus O(H) \oplus O(H)) \rightarrow H^{0}(T, O(3 H)) \rightarrow 0 \\
\| \\
T_{t}(G)
\end{gathered}
$$

Now $d \beta_{a}^{+}=j_{a} i_{a}$, hence $d \beta_{a}^{+}$is injective.

In order to compute the intersection of $T_{a}\left(F^{+}\right)$and $T_{b}\left(F^{+}\right)$in $T_{t}(G)$ one has to recall that the given splitting of $T_{t}(G)$ depends on the ordered choice of $x_{0}, x_{1}, x_{2}, x_{3}$, the equations of the plane $T$. If in the analogous diagram $\left(D_{b}\right)$ we want to give the map $j_{b}$ by means of the natural inclusion of the summands then the diagram is $\left(D_{b}\right)$ :

$$
\begin{gathered}
0 \rightarrow T_{b}\left(F^{+}\right) \stackrel{\iota_{b}}{\rightarrow} H^{0}(A, O(H) \oplus O(H-E) \oplus O(H-E) \oplus O(H-E)) \rightarrow H^{0}(A, O(3 H-2 E)) \rightarrow 0 \\
\quad \downarrow j_{b} \\
H^{0}(T, O(H) \oplus O(H) \oplus O(H) \oplus O(H)) \rightarrow H^{0}(T, O(3 H)) \rightarrow 0 .
\end{gathered}
$$

Next we note that the foldlowing sequence is exact.

$$
\begin{aligned}
0 \rightarrow T_{a}\left(F^{+}\right) \cap T_{b}\left(F^{+}\right) \stackrel{i}{\rightarrow} & H^{0}\left(A, O(H-E)^{\oplus 4}\right) \\
& \quad \stackrel{\left(C_{0}^{-}, C_{1}^{-}, C_{2}^{-}, C_{3}^{-}\right)}{\rightarrow} H^{0}(A, O(3 H-2 E)) \rightarrow 0 .
\end{aligned}
$$

The exactness follows from the proof of (2.20) and more precisely from the remark that one can produce a $L_{3}\left(x_{4}, x_{5}\right)$. The statement about the dimension of the intersection is then obvious. We remark that on the other hand if $C_{0}, C_{1}, C_{2}, C_{3}$ were not linearly independent then the above sequence could not be exact to the right, for simple reasons of rank, so that the analytical branches of $F$ would not be transversal.

(III). In this section we complete the Clemens-Letizia program by proving that the Abel-Jacobi map is not constant on the Fano surface. Let $X$ be a smooth cubic fivefold, we show that the Abel-Jacobi map $a$ : $F \rightarrow J X$ is an immersion at a point $t$ which represents a plane $T$ if $T$ is non-special in the sense of (1.5). Our method follows [10] p. 24.

The cotangent space of $F$ at $t$ is

$$
\left(H^{0}(T, N(T, X))\right)^{*} \simeq H^{2}\left(T, N^{*}(-3)\right) ;
$$

the cotangent space to $J X$ is $H^{2}\left(X, \Omega_{X}^{3}\right)$; the codifferential $a^{*}$ turns out to be the map $k$ in the following commutative diagram, which we explain in 
a moment:

$$
\begin{array}{ccc}
H^{0}\left(X, O_{X}(6) \otimes K_{X}\right) & \stackrel{f}{\rightarrow} & H^{2}\left(X, \Omega_{X}^{3}\right) \\
\downarrow h & & \downarrow k \\
H^{0}\left(T, O_{T}(2)\right) & \stackrel{g}{\rightarrow} & H^{2}\left(T, N^{*}(-3)\right) .
\end{array}
$$

We shall show that $h$ and $g$ are surjective, hence $k$ is also surjective so that $a$ is an immersion at $t$.

The top row is obtained as follows. Start from

$$
0 \rightarrow O(-3)_{X} \rightarrow \Omega_{\mathbf{P}^{6} \mid X}^{1} \rightarrow \Omega_{X}^{1} \rightarrow 0
$$

take then $\wedge^{5}$ and tensor with $O(3)$ so to have

$$
0 \rightarrow \Omega_{X}^{4} \rightarrow \Omega_{\mathbf{P}^{6} \mid X}^{5}(3) \rightarrow \Omega_{X}^{5}(3) \rightarrow 0 .
$$

Taking instead $\wedge^{4}$ one has

$$
0 \rightarrow \Omega_{X}^{3}(-3) \rightarrow \Omega_{\mathbf{P}^{6} \mid X}^{4} \rightarrow \Omega_{X}^{4} \rightarrow 0 .
$$

Putting (3.2) and (3.3) together and tensoring with $O(3)$

$$
0 \rightarrow \Omega_{X}^{3} \rightarrow \Omega_{\mathbf{P}^{6} \mid X}^{4}(3) \rightarrow \Omega_{\mathbf{P}^{6} \mid X}^{5}(6) \rightarrow \Omega_{X}^{5}(6) \rightarrow 0 .
$$

The top row comes from the (hyper)cohomology sequence associated with (3.4).

To find the bottom row in the diagram one starts from the usual sequence of conormal bundles for $\left(T, X, \mathbf{P}^{6}\right)$ :

$$
0 \rightarrow O(-3)_{T} \rightarrow O_{T}(-1)^{\oplus 4} \rightarrow N^{*} \rightarrow 0
$$

taking $\wedge^{3}$ and tensoring with $O(3)$ it follows

$$
0 \rightarrow \Lambda^{2} N^{*} \rightarrow O_{T}^{\oplus 4} \rightarrow \Lambda^{3} N^{*} \otimes O(3) \rightarrow 0 .
$$

Since $c_{1}\left(N^{*}\right)=\Lambda^{3} N^{*}$, then $\Lambda^{3} N^{*} \otimes O_{T}(3)=O_{T}(2)$.

Taking $\Lambda^{2}$ instead one has

$$
0 \rightarrow N^{*}(-3) \rightarrow O_{T}(-2)^{\oplus 6} \rightarrow \wedge^{2} N^{*} \rightarrow 0 .
$$

Putting the sequences together we have the exact sequence

$$
0 \rightarrow N^{*}(-3) \rightarrow O_{T}(-2)^{\oplus 6} \rightarrow O_{T}^{\oplus 4} \rightarrow O_{T}(2) \rightarrow 0 .
$$

The bottom map $g$ in the diagram is obtained by looking at the associated (hyper)cohomology sequence. To check the commutativity of the diagram is now a standard exercise, the main point is to provide a map from the restriction of (3.4) to $T$ to sequence (3.8). We leave the details to 
the reader, everything is based on the commutativity of

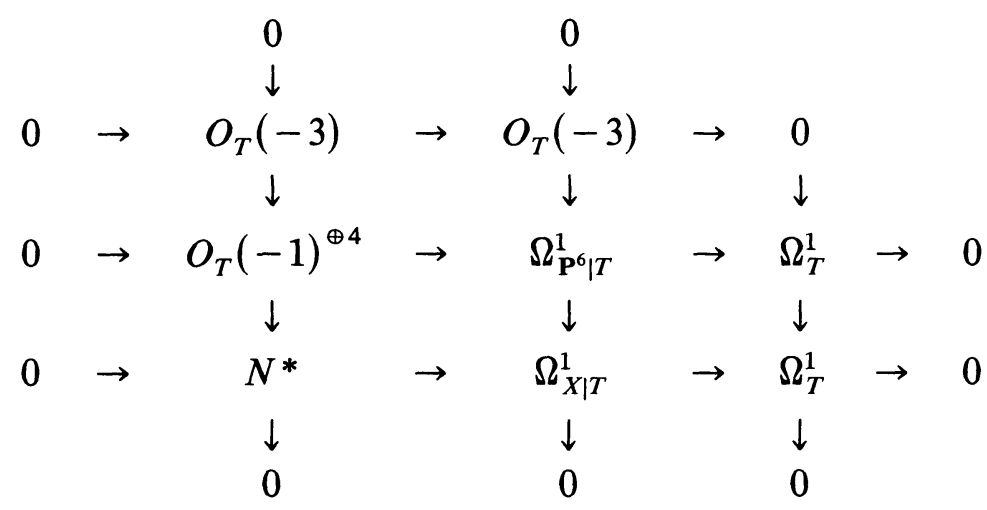

The map $h$ in the diagram is obviously surjective, being the restriction map. The surjectivity of $g$ is a simple consequence of the vanishing $h^{2}\left(O_{T}(-2)\right)=0=h^{1}\left(O_{T}\right)$.

\section{REFERENCES}

[1] A. B. Altman and S. L. Kleiman, Foundations of the theory of Fano schemes, Compositio Math., 34 (1977), 3-47.

[2] W. Barth and A. Van de Ven, Fano varieties of lines on hypersurfaces, Arch. Math., 31 (1978), 96-104.

[3] S. Bloch and J. P. Murre, On the Chow group of certain types of Fano threefolds, Compositio Math., 39 (1979), 47-105.

[4] H. Clemens, On the surjectivity of the Abel-Jacobi mappings, Annals of Math., 117 (1983), 71-76.

[5] H. Clemens and P. A. Griffiths, The intermediate Jacobian of the cubic threefold, Annals of Math., 95 (1972), 281-356.

[6] A. Collino, J. P. Murre and G. E. Welters, On the family of conics lying on a quartic threefold, Rend. Sem. Mat. Univers. Politecn. Torino, 38 (1980), 151-181.

[7] P. Deligne and N. Katz, Groupes de Monodromie en Géométrie Algébrique, SGA 7 II, Springer Lecture Notes 340, 1973.

[8] P. Griffiths, Complex analysis and algebraic geometry, Bull. Amer. Math. Soc., 1 (1979), 595-626.

[9] M. Letizia, The Abel-Jacobi mapping for the quartic threefold, Invent. Math., 75 (1984), 477-492.

[10] G. E. Welters, Abel-Jacobi isogenies for certain types of Fano threefolds, Mathematisch Centrum Amsterdam, 1981.

Received May 25, 1984. Supported in part by grants from the Italian Ministry of Public Education and the C. N. R. 



\section{PACIFIC JOURNAL OF MATHEMATICS EDITORS}

\author{
V. S. VARADARAJAN (Managing Editor) \\ University of California \\ Los Angeles, CA 90024 \\ Hebert Clemens \\ University of Utah \\ Salt Lake City, UT 84112 \\ Charles R. DePrima \\ California Institute of Technology \\ Pasadena, CA 91125
}

R. FINN

Stanford University

Stanford, CA 94305

HermanN FlaschKa

University of Arizona

Tucson, AZ 85721

RAMESH A. GANGOLlI

University of Washington

Seattle, WA 98195

ROBION KIRBY

University of California

Berkeley, CA 94720

ASSOCIATE EDITORS
R. ARENS
E. F. BECKENBACH
B. H. NEUMANN
F. WOLF
K. YoshidA (1906-1982)

C. C. Moore

University of California

Berkeley, CA 94720

H. SAMELSON

Stanford University

Stanford, CA 94305

HAROLD STARK

University of California, San Diego

La Jolla, CA 92093

\section{SUPPORTING INSTITUTIONS}

UNIVERSITY OF ARIZONA

UNIVERSITY OF BRITISH COLUMBIA

CALIFORNIA INSTITUTE OF TECHNOLOGY

UNIVERSITY OF CALIFORNIA

MONTANA STATE UNIVERSITY

UNIVERSITY OF NEVADA, RENO

NEW MEXICO STATE UNIVERSITY

OREGON STATE UNIVERSITY
UNIVERSITY OF OREGON

UNIVERSITY OF SOUTHERN CALIFORNIA

STANFORD UNIVERSITY

UNIVERSITY OF HAWAII

UNIVERSITY OF TOKYO

UNIVERSITY OF UTAH

WASHINGTON STATE UNIVERSITY

UNIVERSITY OF WASHINGTON 


\section{Pacific Journal of Mathematics}

Vol. 122, No. $1 \quad$ January, 1986

Michael James Cambern, Near isometries of Bochner $L^{1}$ and $L^{\infty}$ spaces ....1 Kun Soo Chang, Gerald William Johnson and David Lee Skoug, The

Feynman integral of quadratic potentials depending on two time

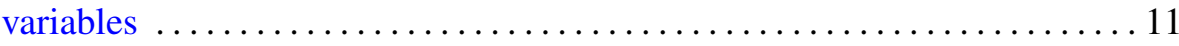

Robert Coleman, One-dimensional algebraic formal groups $\ldots \ldots \ldots \ldots \ldots 35$

Alberto Collino, The Abel-Jacobi isomorphism for the cubic fivefold .......43

N. J. Dev and S. S. Khare, Finite group action and vanishing of $N_{*}^{G}[F] \ldots 57$

Harold George Diamond and Jeffrey D. Vaaler, Estimates for partial sums

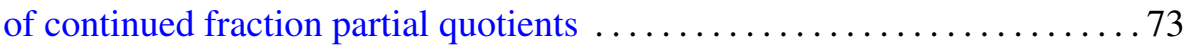

Kenneth R. Goodearl, Patch-continuity of normalized ranks of modules

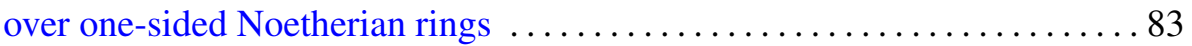

Dean Robert Hickerson and Sherman K. Stein, Abelian groups and

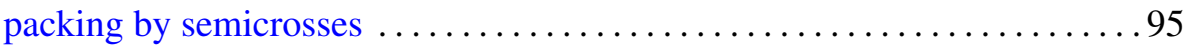

Karsten Johnsen and Harmut Laue, Fitting structures $\ldots \ldots \ldots \ldots \ldots 11$

Darren Long, Discs in compression bodies . ................... 129

Joseph B. Miles, On the growth of meromorphic functions with radially distributed zeros and poles ........................... 147

Walter Volodymyr Petryshyn, Solvability of various boundary value problems for the equation $x^{\prime \prime}=f\left(t, x, x^{\prime}, x^{\prime \prime}\right)-y \ldots \ldots \ldots \ldots \ldots . \ldots 169$

Elżbieta Pol, The Baire-category method in some compact extension problems

Masami Sakai, A new class of isocompact spaces and related results 211

Thomas Richard Shemanske, Representations of ternary quadratic forms and the class number of imaginary quadratic fields ..

Tsuyoshi Uehara, On class numbers of cyclic quartic fields 